\title{
BMJ Open Older doctors and progression through specialty training in the UK: a cohort analysis of General Medical Council data
}

\author{
Yvette Pyne, ${ }^{1}$ Yoav Ben-Shlomo ${ }^{2}$
}

To cite: Pyne $Y$, Ben-Shlomo Y. Older doctors and progression through specialty training in the UK: a cohort analysis of General Medical Council data. BMJ Open 2015;5:e005658. doi:10.1136/bmjopen-2014005658

- Prepublication history and additional material is available. To view please visit the journal (http://dx.doi.org/ 10.1136/bmjopen-2014005658)

Received 11 May 2014 Revised 13 October 2014 Accepted 15 October 2014

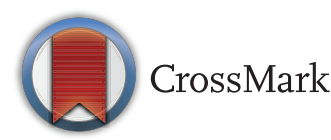

\footnotetext{
${ }^{1}$ University of Bristol Medical School, Bristol, UK

${ }^{2}$ School of Social and Community Medicine, Bristol, UK
}

Correspondence to Ms Yvette Pyne; yvette.pyne@doctors.org.uk

\section{ABSTRACT}

Objective: To determine whether older age at graduation is associated with any difference in outcomes from the annual specialty training progression assessment.

Design: An open cohort of 38308 doctors who graduated from a UK medical school with annual assessments of progression in their specialty training programme with data centrally collected by the General Medical Council between 5 August 2009 to 31 July 2012.

Results: Mature junior doctors ( $\geq 29$ years at graduation) were more likely to have problems with progression on their annual review of competence progression record of in training assessment (ARCP/RITA) than their younger colleagues (OR 1.34, $95 \% \mathrm{Cl} 1.22$ to $1.49, \mathrm{p}<0.001)$. This association was, if anything, even stronger (OR $1.57,95 \% \mathrm{Cl} 1.41$ to 1.74 , $p<0.001$ ) after adjustment for gender, ethnicity, type of University and specialty. The same was true when only looking at the most extreme ARCP outcome (4) which is being asked to leave their specialist programme ( $O R$ $1.81,95 \% \mathrm{Cl} 1.34$ to $2.44, \mathrm{p}<0.001$ ).

Conclusions: Mature doctors are a growing part of the medical workforce and they are likely to broaden the spectrum of doctors by bring different life experience to the profession. These results suggest that they are more likely to have problems with progressing through their specialist training programme. More research is required to determine the reasons behind these associations and how mature doctors can be supported both in choosing the best training programme and in coping with the complex demands of higher training at a later stage in their lives.

\section{INTRODUCTION}

Over the past decade, more mature students have been welcomed onto the medical training programme. While they only make up around $4 \%$ of medical students in the UK, ${ }^{1}$ they are a more substantial proportion of graduates from the USA and Canada $(16.7 \%$ and $14.2 \%$ were 30 or older at graduation, respectively ${ }^{2}{ }^{3}$ ). These students are often

\section{Strengths and limitations of this study}

- First study to look at how age at graduation affects a doctor's chance of succeeding in their annual revalidation.

- Large sample size with little missing data and minimal sources of bias for exposure and outcome variables.

- Results are counter to prevailing beliefs that mature medical students cope better with medical training as demonstrates greater problems with progression through the annual review of competence progression (ARCP) process.

- Highlights the importance of other demographic and clinical factors that determine progression in training.

- ARCP data is a simple measure of adequate progression and does not capture excellence so could hide a bimodal distribution whereby mature junior doctors are also more likely to excel as well as have problems of progression.

different in their outlook and abilities to a typical school leaver and may be better suited as both a student and future doctor. For example, the former director of the graduate entry programme at St George's Hospital Medical School has stated that 'mature students... are sooner and better able to handle the responsibilities of being a doctor' and are 'much more self-directed, challenging, demanding, questioning, and mature' than their younger counterparts. ${ }^{4}$ These subjective views have some limited support from both qualitative and quantitative research during the medical school years, for example, older students appear to do better at year 3 Objective Structured Clinical Examination exams. $^{5}$ Two studies have suggested that mature students cope better with the transition to clinical responsibilities feeling less confused, daunted, anxious or intimidated and more likely to describe a positive transition. ${ }^{6} 7$ This may not merely reflect greater 
academic experience; greater age at programme entry, as opposed to the presence of a previous degree, was a better predictor for positive attributes and attitudes related to being a doctor. ${ }^{8}$ This may reflect stronger motivational factors that lead them to positively choose medicine as a subsequent career.

Remarkably little is known about what happens to these mature graduates after they qualify. These positive attitudes could result in very focused and determined graduates who try to reach their choice of specialist career as quickly and efficiently as possible thereby progressing through their training rapidly. On the other hand, mature graduates are more likely to have established geographical roots and family commitments that may make handling the double burdens of career and family problematic even earlier in their training as compared to younger graduates. Anecdotal evidence from the Severn Deanery has suggested that some mature students required greater support with getting through their annual assessment (previously known as RITArecord of in training assessment) and now referred to as ARCP (annual review of competence progression). We objectively test the null hypothesis that the proportion of doctors who either require additional training time or who are asked to leave the programme is the same for both older and younger graduate doctors.

\section{METHODS}

\section{Datasource and variable definitions}

The General Medical Council (GMC), who collate the national data on ARCP/RITA, kindly provided us with an anonymised extract of data for all UK medical graduates who had a review between 5 August 2009 to 31 July 2012. In the UK, prior to 2013, the ARCP/RITA process begins at the start of specialty training (such as surgery or primary care) and continues until completion of training (obtaining a certificate of completion of training-CCT) that enables doctors to apply for a consultant post.

Since the coding of the outcomes for ARCP and RITA do not map directly onto each other, we had to use slightly different definitions for our outcome measure of poor progression. For ARCP we used codes 3 (requires additional training time), 4 (released from the programme) and 7.3 (inadequate progress) as a composite measure of poor progression. For the RITA we used codes D (targeted training) and $\mathrm{E}$ (intensified or repeat training) as our poor outcome measure (see online supplementary appendix 1 for the full coding scheme).$^{9} 10$ We choose to exclude participants with a code for insufficient evidence (as this often reflects inadequate documentation rather than poor progress per se) and those trainees on an out-of-programme secondment.

Our exposure measure was based on an arbitrary age cut-off (coded as an integer value). There is no accepted standard definition of a 'mature' student so we chose to define this as a graduate who was 29 years or over at the year of first registration (ie, year of graduation). By choosing this cut point we hoped to not include graduates who had simply taken a gap year, intercalated BSc or a prior degree before going straight into medicine (as this should mean they are not older than 27 years) but those who would have had some years of 'work' experience outside of medicine. This is similar to a previous study that defined the 'older mature' as 'students who have worked in other occupations for a number of years prior to making a decision to apply to medical school ${ }^{11}$ We further subdivided this 'mature' group into those aged between 29 and 31 years and those who were 32 years or older on date of first registration to examine for any dose-response effects with older age at registration. Finally for a sensitivity analysis we examined a more detailed classification of the younger baseline group into the following categories $(\leq 23,24,25,26,27,28$ years). We defined, a priori, a number of potential confounders or intermediaries that could be associated with being an older graduate and a greater probability of poor progression. These were gender, specialty, ethnicity and whether the graduate had qualified from a 'mature friendly' medical school that may be better able to help the older graduate cope with the future stresses of being a doctor. This last variable was operationalised as follows: We calculated the percentage of mature students graduating from the medical school and then created a binary variable if the percentage was greater than $10 \%$-approximately the top quartile and these were mainly the new medical schools (eg, Exeter, Brighton \& Sussex etc). We could not disaggregate all the London-based medical schools as they were all coded as University of London.

\section{Statistical methods}

The original data set had multiple records for a doctor for each assessment (long format) but this could be linked by an anonymous unique identifier. We reshaped the data into wide format (one row per doctor) so each doctor is only represented once in the data set. If the doctor had poor progression more than once, we only coded the first event. We compared simple proportions using $\chi^{2}$ tests and linear regression for continuous variables. We then calculated the crude OR (95\% CI, p values) for older age at graduation and poor progression and multivariable $\mathrm{OR}$ adjusting for gender, ethnicity (binary variable defined as non-ethnic if ticked any of the White ethnicity codes from census or ethnic minority, which included any other code), specialty (dummy variable) and mature friendly medical school (binary variable). For specialty we used hospital medicine as the baseline group as it had the largest number of doctors. We undertook a sensitivity analysis using the most extreme outcome-leaving the training programme. As this is only explicitly coded in the ARCP outcomes, we could not use participants with RITA assessments for this secondary analysis. We examined for potential interactions between age at registration with gender and ethnicity and either failure to progress or being asked to leave the specialty. 


\section{RESULTS}

We received a total of 110571 records (multiple assessments per doctor). We dropped 307 records (0.3\%) without a specialty code and there were 5173 records with a missing outcome $(4.7 \%)$ and 361 records $(0.3 \%)$ with an ambiguous code that we could not use (99\% of the missing outcome data came from 2012, when the GMC asked Deaneries to return forms even for doctors who were not having ARCPs as they were out of programme, on maternity leave or long-term sick so these are not really missing outcomes-Andy Knapton, GMC personal communication). In addition, there were 7072 records (6.4\%) for out-of-programme secondments and 7737 records (7\%) coded as insufficient evidence leaving us with 89921 records. After removing incomplete data for ethnicity, year of birth, year of registration and graduating university, we were left with 83702 records from 38308 doctors (see figure 1) similar to the stated number of registered doctors (in approved practice settings) as listed by the GMC. ${ }^{12}$ There were $2610(6.8 \%)$ mature graduates (1414 between 29 and 31 years, and $1196 \geq 32$ years); $83.7 \%$ of assessments were ARCP and $16.3 \%$ were from the RITA. In total, 6045 doctors $(15.8 \%)$ failed at least one ARCP or RITA during the 3 years of recorded data and of those, 491 (1.3\%) were asked to leave the specialty programme (ARCP outcome 4).

Older doctors were more likely to be male, non-ethnic minority, and train in primary care or public health $(\mathrm{p}<0.001)$ compared to younger doctors (see web table 1$)$. Older doctors were more likely to have problems with progression (OR 1.34, 95\% CI 1.22 to 1.49 , p value $<0.001$; table 1). After adjusting for gender, ethnicity, type of medical school, and choice of specialty, the OR was further increased (OR 1.57, 95\% CI 1.41 to 1.74 , $\mathrm{p}<0.001$ ). When we broke down the older age group into three categories (non-mature, 29 to 31 years, $\geq 32$ years), the trend was even more marked both with and without adjustment for other covariates (OR 1.0, 1.43, 1.74, respectively, $p$ value for trend $<0.001$ after multivariable adjustment). Our more detailed breakdown of the younger age group suggested that increased problems with progression are evident at a younger age, 26 years and above, though the oldest group ( $\geq 32$ years) appear to have additional problems (see web table 2).

Our secondary analysis using just the extreme outcome of leaving the training programme (ARCP-4) found an even greater OR of failing to progress for mature students compared to non-matures (OR 1.81, 95\% CI 1.34 to 2.44, $\mathrm{p}<0.001)$. When we examined this by our three level age group, we observed a non-linear trend (OR for nonmature, 29 to 31 years, $\geq 32$ years: $1.0,1.29,2.48$, respectively, $\mathrm{p}$ value for trend $<0.001$ ) whereby the excess risk seemed mainly limited to the oldest group ( $\geq 32$ years; see web table 3). There was no evidence of any interactions between maturity and either gender or ethnicity on failure to progress or being asked to leave the specialty. The results were essentially unchanged when we replaced the type of university with a dummy variable for all universities.

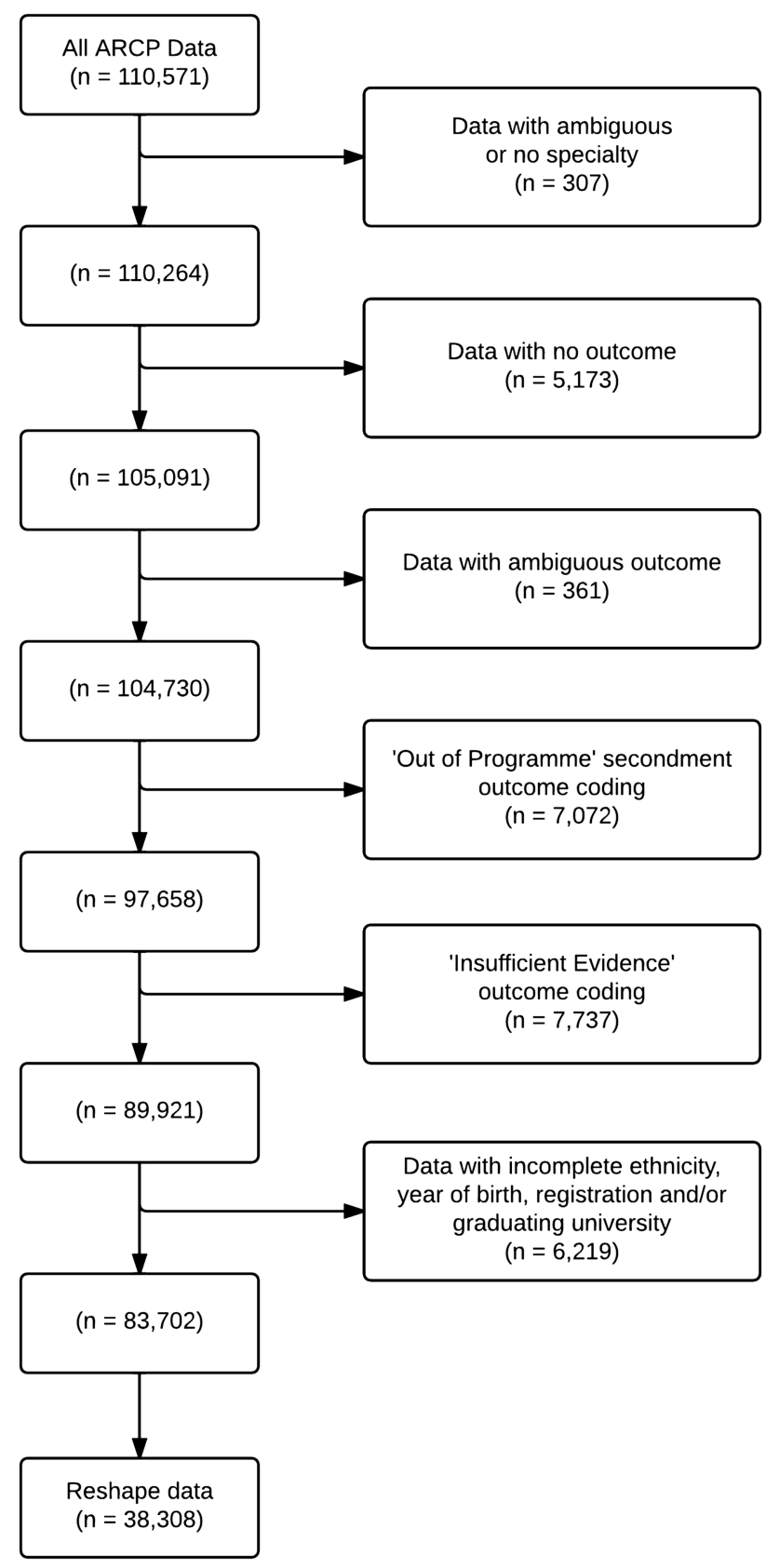

Figure 1 A flow chart showing losses of data due to incomplete or inadequate data to reach the final study sample.

\section{DISCUSSION}

This study provides strong evidence that doctors who are older at graduation were more likely to have problems with progression at their annual assessment and were more likely to leave their initial specialist training programme. These findings appeared to be independent of other factors, such as gender, ethnicity, type of medical school and specialty. The last showed wide variability with some specialties having higher (obstetrics and gynaecology) and others lower (general practice and public health) rates of problems with progression. This finding is consistent with the results of a recent analysis comparing doctors who obtained their medical degree 
Table 1 Association between 'Mature status' and failure to progress at ARCP adjusted for a range of potential confounders

\begin{tabular}{|c|c|c|c|c|}
\hline & Model 1* OR (95\% Cl) & p Value & Model 2* OR (95\% Cl) & p Value \\
\hline Older ( $\geq 29$ years) $(2610)$ & 1.34 (1.22 to 1.49$)$ & $<0.001$ & $1.57(1.41$ to 1.74$)$ & $<0.001$ \\
\hline Younger group ( $\leq 28$ years) (35 698) & 1.00 & & 1.00 & \\
\hline Older group (29-31 years) (1414) & 1.27 (1.11 to 1.46$)$ & 0.001 & $1.43(1.24$ to 1.65$)$ & $<0.001$ \\
\hline Oldest group ( $\geq 32$ years) (1196) & $1.43(1.24$ to 1.65$)$ & $<0.001$ & 1.74 (1.50 to 2.02$)$ & $<0.001$ \\
\hline $\mathrm{p}$ Value for trend & & $<0.001$ & & $<0.001$ \\
\hline Female gender (21 470) & & & $0.82(0.77$ to 0.87$)$ & $<0.001$ \\
\hline Ethnic minority $(11338)$ & & & 1.59 (1.49 to 1.68$)$ & $<0.001$ \\
\hline Mature friendly university (35 745) & & & $1.18(1.06$ to 1.32$)$ & 0.003 \\
\hline \multicolumn{5}{|l|}{ First specialty } \\
\hline Medicine (10 135) & & & 1.00 & \\
\hline ACCS and related (5827) & & & 1.00 (0.92 to 1.08$)$ & 0.93 \\
\hline Surgery (6077) & & & $0.84(0.77$ to 0.91$)$ & $<0.001$ \\
\hline GP and public health (9094) & & & $0.26(0.24$ to 0.29$)$ & $<0.001$ \\
\hline$O$ and $G(1528)$ & & & 2.16 (1.91 to 2.43$)$ & $<0.001$ \\
\hline Paediatrics (2791) & & & 0.81 (0.72 to 0.90$)$ & $<0.001$ \\
\hline Pathology (564) & & & $0.84(0.67$ to 1.06$)$ & 0.14 \\
\hline Psychiatry (966) & & & 0.51 (0.42 to 0.63$)$ & $<0.001$ \\
\hline Radiology (1326) & & & 0.88 (0.76 to 1.02$)$ & 0.10 \\
\hline
\end{tabular}

${ }^{*}$ Model 1, simple OR; Model 2 for binary age group after adjustment for all covariates as shown in table except for the three level age group variable. This model was then rerun with the three level age group and other covariates to examine for a dose-response effect. ACCS, acute care common stem; GP, general practitioner; $\mathrm{O}$ and $\mathrm{G}$, obstetrics and gynaecology.

either in or outside of the UK and testing whether the Performance and Linguistics Assessments Board examination system explained performance at ARCP. ${ }^{13}$ It is also consistent with the recent GMC Report on the state of medical education and practice in the $\mathrm{UK}^{14}$ which found (in Figure 46) that doctors who were over 30 when joining the register were more likely than their younger counterparts to receive a sanction or a warning. While the null hypothesis defined 'mature' graduates as those over 28 years at first registration, additional analysis has highlighted this effect is evident for doctors as young as 26 on registration, who make up over $20 \%$ of the doctor population in this sample.

As these results are unlikely to be due to chance, one must consider other possible explanations. Bias in either measurement of exposure or outcome is very unlikely as age at graduation is taken from year of registration and year of birth so should be well recorded and any coding errors are likely to be random in nature. Similarly any coding errors in the ARCP/RITA outcomes are unlikely to be differential according to age at graduation. A very small proportion of outcome data were missing and this is unlikely to have been systematically biased. Though we attempted to control for a variety of covariates that could influence the outcome, we did not have reliable data on whether trainees were in full or part time training. The latter may be more common in mature graduates and may influence progression in training. Similarly we could not explore if there was an interaction between mature graduate status and full-time or part-time training. In addition, ARCP is not intended to capture excellence in training but merely adequate progression. It is possible that the performance of mature graduates is bimodal so that some mature doctors actually have better outcomes but this would not be evident in our analysis.

One must consider several possible explanations as to why older graduates have more problems progressing through higher training if we assume our observed associations are truly causal. (1) They may have more commitments outside of work (caring commitments for either children or parents or other personal relationship issues) that may make it harder to successfully complete all the assessments required for ARCP. ${ }^{11}$ (2) They may find themselves committing to a specialty that may not have been their first choice in order to stay in a certain part of the country for their children or spouse. This could result in them doing less well in ARCP due a degree of ambivalence to this specialty. (3) They may have more problems passing postgraduate specialist exams or completing more technical skills competencies which result in either additional training time or in the worst case leaving the specialty. This may be one explanation why we observed the same difficulty with progression for doctors of ethnic minority background who are known to have a higher failure rate with the Membership of the Royal College of General Practitioners (MRCGP) exam. $^{13}{ }^{15}$ (4) Being older, these doctors may find it harder to engage with the informal social support groups among junior doctors (either due to personal commitments or the age gap) and thus have fewer resources to call on during challenging rotations or clinical situations. (5) The higher rate of leaving the programme in the oldest age group may reflect an inappropriate choice of specialty or that older graduates, having had a past career and already made one major change, have more confidence to switch specialties than younger graduates. 
These results should not be interpreted as older graduates are therefore less competent doctors. The ARCP/ RITA assessments are there to monitor training progression against specific competencies and milestones and are not a direct measure of the quality of doctors. Some excellent doctors simply take longer to complete their training and may have gained additional skills and life experiences on this journey, learning more from their mistakes than their successes.

These results, however, should not be a cause for complacency. Longer training programmes exert additional financial pressures on training budgets and any doctor who leaves medicine altogether at this stage has had a lot of time and money invested into their training. The problem is not unique to older graduates as we observed that men, ethnic minorities and some specialties showed the same pattern of results.

In conclusion, mature doctors are part of the makeup of the NHS workforce and they widen the variety of doctors as well as bringing insights from past careers that is to be welcomed. While they appear to do better than their younger counterparts at university, they are more likely to have problems with specialty training in the UK. We believe that the causes for this are multifactorial and probably not unique to the UK but generalisable to other high-income countries like the USA and Canada, though this requires empirical confirmation. These results should be an impetus for further qualitative research to provide greater insights into why older graduates are more like to have difficulties in progression and direct action from training programmes so that they can identify problems at an earlier stage and provide greater support for such trainees as appropriate.

Acknowledgements The author would like to acknowledge Andy Knapton and Daniel Smith at the General Medical Council (GMC) for their support, guidance and encouragement and the GMC for providing us the data for our research. Finally, the authors would thank Dr Clare Van Hamel, Associate Postgraduate Dean and Director of Severn Deanery Foundation School for her anecdotal comments that stimulated this project.

Contributors YP conceived of the idea for this piece of work as her student SSC and approached the GMC for data access. YB-S acted as her SSC supervisor and provided support and training for the statistical analysis. YP undertook the initial data cleaning and analysis. YB-S checked the analyses and undertook some additional analyses. YP drafted the first version of the paper that was then edited by YB-S. All authors approved the final version of the manuscript. YP acts as the guarantor.

Funding This research received no specific grant from any funding agency in the public, commercial or not-for-profit sectors.

Competing interests YB-S regularly sits on ARCP committees for Public Health trainees.
Ethics approval The authors did not seek formal NHS ethical approval for this study as it was a secondary data analysis of existing data that had been anonymised to ensure data confidentiality.

Provenance and peer review Not commissioned; externally peer reviewed.

Data sharing statement No additional data are available.

Open Access This is an Open Access article distributed in accordance with the Creative Commons Attribution Non Commercial (CC BY-NC 4.0) license, which permits others to distribute, remix, adapt, build upon this work noncommercially, and license their derivative works on different terms, provided the original work is properly cited and the use is non-commercial. See: http:// creativecommons.org/licenses/by-nc/4.0/

\section{REFERENCES}

1. Higher Education Statistics Agency. Medicine Undergraduates Data 2012-2013.

2. Association of American Medical Colleges. Medical School Graduation Questionnaire-2013 All Schools Summary. 2013.

3. Association of American Medical Colleges. Canadian Medical School Graduation Questionnaire-2012 All Schools Summary Report. 2012.

4. McCrorie P. Graduate students are more challenging, demanding, and questioning. BMJ 2002;325:676. http://www.pubmedcentral.nih. gov/articlerender.fcgi?artid=1124216\&tool=pmcentrez\&rendertype= abstract (accessed 18 Jun 2013).

5. Lumb AB, Vail A. Comparison of academic, application form and social factors in predicting early performance on the medical course. Med Educ 2004;38:1002-5.

6. Shacklady J, Holmes E, Mason G, et al. Maturity and medical students' ease of transition into the clinical environment. Med Teach 2009;31:621-6.

7. Hayes K, Feather A, Hall A, et al. Anxiety in medical students: is preparation for full-time clinical attachments more dependent upon differences in maturity or on educational programmes for undergraduate and graduate entry students? Med Educ 2004;38:1154-63.

8. Wilkinson TJ, Wells JE, Bushnell JA. Are differences between graduates and undergraduates in a medical course due to age or prior degree? Med Educ 2004;38:1141-6.

9. Annual Review of Competence Progression (ARCP)-Severn Deanery-NHS. http://www.severndeanery.nhs.uk/about/ education-and-training/doctors-in-training/annual-review-ofcompetence-progression-arcp/ (accessed 20 Jun 2013).

10. MMC. The gold guide: a reference guide for postgraduate specialty training in the UK. 4th edn. Health Education England, 2010.

11. Mathers J, Parry J. Older mature students' experiences of applying to study medicine in England: an interview study. Med Educ 2010;44:1084-94.

12. List of Registered Medical Practitioners-statistics. http://www. gmc-uk.org/doctors/register/search_stats.asp (accessed $15 \mathrm{Jul}$ 2013).

13. Tiffin PA, Illing J, Kasim AS. Annual Review of Competence Progression (ARCP) performance of doctors who passed Professional and Linguistic Assessments Board (PLAB) tests compared with UK medical graduates : national data linkage study. BMJ 2014;2622:1-18.

14. General Medical Council. The state of medical education and practice in the UK 2014. 2014. http://www.gmc-uk.org/SOMEP_ 2014.pdf_58053580.pdf

15. Esmail A, Roberts C. Academic performance of ethnic minority candidates and discrimination in the MRCGP examinations between 2010 and 2012: analysis of data. BMJ 2013;347:f5662. 